\title{
ENTRE "LOS PLACERES DEL EXILIO" Y LOS DESCONTENTOS DE LA MIGRACIÓN: LUCY, NOVELA DE JAMAICA KINCAID ${ }^{1}$
}

La distancia con respecto al país natal juega un rol muy importante en las narrativas de autores exiliados o migrantes. Independientemente de la valoración que se le otorgue en cada narrativa, la distancia se asocia a una serie de tópicos que han permitido a la crítica literaria distinguir una "estética del exilio". Así, por ejemplo, Edward Said (2003) habla de la visión en contrapunto que se desarrolla con la experiencia exílica, la cual aportaría una visión aguzada y con mayor distancia crítica tanto en relación al lugar de origen como al país de recepción. El exilio favorecería el cuestionamiento de la naturalización de las perspectivas, asociada a la permanencia en un solo lugar. El exiliado, al estar permanentemente confrontado a distintas maneras de ver, hacer y sentir las cosas, tendría la posibilidad de ampliar sus horizontes de comprensión e interpretación de las relaciones sociales y las experiencias de vida. Estas son algunas de las facetas positivas que el escritor de Barbados, George Lamming, le atribuye al exilio en su clásica colección de ensayos The Pleasures of Exile (1960), que parafraseamos en el título de este artículo.

En relación a la literatura caribeña contemporánea - y más específicamente a la emergencia de la literatura del Caribe anglófono- Belinda Edmonson (1999) habla de dos generaciones de escritores claramente diferenciadas en función del destino de emigración y de la composición de género de sus autores. Carine M. Mardorossian (2002) retoma esta distinción y la hace extensiva al Caribe francés. La primera generación de escritores del Caribe anglófono y francófono se formó en las metrópolis coloniales - Londres y París, respectivamente - que en la primera mitad del siglo $\mathrm{XX}$ se convirtieron en lugares de encuentro de autores ansiosos por acceder al tipo de autoridad literaria asociada con Cambridge y Oxford (Edmonson, 1999) y a las oportunidades editoriales y de difusión de las que carecían en las islas de origen (Low 2002). Los exiliados formaban parte, por lo general, de ese sector de las sociedades coloniales que recibía una educación occidental que buscaba "asimilarlos" a la cultura metropolitana y del que se esperaba que actuara como intermediario entre el poder colonizador y la masa de colonizados. Sin embargo, en la metrópolis, junto con posibilidades de desarrollar una carrera

\footnotetext{
${ }^{1}$ Resultado de la investigación realizada en el marco del Proyecto Fondecyt de Iniciación No 11080065 , dirigido por la autora.
} 


\section{Lucía Stecher Guzmán}

literaria, estos autores se encontraron, también, con el rechazo de la sociedad metropolitana a considerarlos tan "europeos" como su educación isleña les había enseñado que podían ser. Por otra parte, en Europa establecieron contactos e intercambios con otros intelectuales pertenecientes al mundo colonizado y a movimientos que afirmaban una común identidad panafricana (como el renacimiento de Harlem y el indigenismo haitiano de las primeras décadas del siglo XX). Así, la emergencia de esta primera generación de escritores del Caribe anglófono y francófono está asociada al encuentro metropolitano de antillanos y miembros de la diáspora africana, unidos en un primer momento por el proyecto común de reivindicar la raza negra (pensemos sobre todo en la negritud del martiniqueño Aimé Cesaire, el senegalés Leopold Senghor y el guyanés Léon Damas) y, posteriormente, por un ideario político nacionalista que los comprometía en la lucha por la descolonización de sus países natales.

Los textos literarios producidos por esta primera generación de escritores exiliados se caracterizan, según Mardorossian, por la construcción de mundos de ficción que representan en términos dicotómicos las relaciones entre el país de origen y el del exilio. El primero aparece como un espacio añorado, romantizado, idealizado, mientras el segundo es representado como un lugar hostil y alienante (salvo en el caso de V.S. Naipaul quien, si bien tiende también a la construcción de oposiciones binarias, suele invertir la valoración otorgada a cada uno de los espacios). El origen, por lo general, se asocia a la tradición y a la continuidad de las experiencias culturales, mientras el destino del exilio aparece representando una modernidad e impulsos modernizadores connotados negativamente. En la "estética del exilio", el retorno aparece siempre en el horizonte de los deseos y posibilidades de personajes quienes viven añorando la reincorporación a su tierra natal.

En el caso de lo que Belinda Edmonson denomina la segunda generación de autores del Caribe anglófono - compuesta principalmente por mujeres que migraron a Estados Unidos por razones económicas y que desarrollaron su vocación literaria con posterioridad a la emigración-, críticas como Mardorossian (2002) y Jurney (2009) describen la configuración de una nueva estética que estaría dejando atrás las articulaciones dicotómicas asociadas a la escritura del exilio. Esto se traduciría en una perspectiva distinta para abordar las relaciones que se establecen entre el "país de origen" y el "país de destino". Mardorossian habla de la "literatura migrante" que desafía "la lógica binaria al enfatizar el movimiento, el desarraigo y la mezcla de culturas, razas y lenguajes" (16, todas las traducciones del inglés al castellano son mías) y que se caracteriza por mayores niveles de ambigüedad y de ambivalencia en la representación de los sentimientos y relaciones de los personajes con respecto a los mundos que habitan y han dejado atrás. Jurney, por su parte, 
propone el término de "literatura diaspórica" para dar cuenta de estas nuevas narrativas que configuran espacios transnacionales y fluidos, concebidos, sobre todo, en términos de comunidades de personas más que de territorios geográficos claramente delimitados. Para Jurney, el término "diaspórico" da cuenta de mejor manera que el propuesto por Mardorossian, de los orígenes e historias particulares de quienes deciden o se ven obligados a emigrar.

Ambas críticas coinciden, sin embargo, en que no todas las narrativas producidas por autoras caribeñas migrantes caben dentro de las categorías de "migrantes" o "diaspóricas". Mardorossian advierte sobre la necesidad de evitar la tendencia a fundamentar las lecturas críticas de obras literarias en la categoría de la experiencia. Es decir, una similar experiencia de exilio o migración no tiene por qué traducirse en estéticas o políticas de representación equivalentes en distintos autores. Así, Mardorossian muestra que si bien tanto Edwidge Danticat como Julia Álvarez son caribeñas que viven y escriben desde Estados Unidos novelas basadas en gran medida en su propia experiencia migratoria, la primera sigue produciendo dentro de la estética nacionalista asociada a la primera generación de escritores caribeños, mientras que la obra de la segunda forma parte de la naciente "literatura migrante". Mardorossian parece considerar anacrónica la propuesta narrativa de Danticat, mientras celebra el carácter deconstructivo de las novelas de Álvarez, y más específicamente de Yo (1997).

Mardorossian sostiene que Breath, Eyes, Memory (Palabra, Ojos, Memoria, 1994) de la escritora haitiana-americana Edwidge Danticat se sustenta sobre una serie de oposiciones binarias que remiten a la estética del exilio. Los principales términos dicotómicos serían el país de origen (Haití) y el de destino (Estados Unidos) —representativos de un pasado idílico y de un presente conflictivo, respectivamente- y entre tradición y modernidad. La defensa del mundo tradicional haitiano - aun cuando se critiquen sus rasgos machistas y opresivos - estaría asociada principalmente, en la novela de Danticat, a la conservación y recomposición de lazos al interior de genealogías de mujeres. La crítica que realiza Mardorossian a esta propuesta de Danticat coincide con la observación de Katherine Sugg (2002) sobre la tendencia de algunos textos postcoloniales y análisis feministas a realzar las dimensiones comunitarias y de celebración del arraigo en las narrativas de mujeres por sobre las propuestas de separación y ruptura de lazos familiares y culturales. Las mujeres, así, continuarían siendo las responsables de la continuidad de los lazos familiares y culturales, a la vez que estarían especialmente ligadas y asociadas a la tierra de origen, a las raíces. Además de mantener estas asociaciones de signo patriarcal — aunque en el discurso feminista liberal sean connotadas positivamente- estas lecturas tienden a desconocer las diferencias de clase, raza y origen que hacen de "las mujeres" una entidad 


\section{Lucía Stecher Guzmán}

muy heterogénea y diferenciada. Por otra parte, ofrecen poco espacio para aquellas narrativas de mujeres que reivindican el derecho a la ruptura de vínculos comunitarios y a explorar nuevas posibilidades de construir su historia personal.

Lucy, la narradora protagonista de la novela epónima de Jamaica Kincaid, es uno de los personajes femeninos más atípicos y rupturistas de la narrativa contemporánea de autoras caribeñas residentes en Estados Unidos. La nostalgia, la distancia, la (im)posibilidad del retorno, así como las relaciones entre mujeres y con la comunidad de origen, son narradas en el texto de Kincaid con un tono irónico y dolorosamente descreído. Si bien a primera vista podría identificarse la obra de Kincaid con la estética migrante o diaspórica propuestas por Mardorossian y Jurney, respectivamente, me parece que $L u c y$,en particular, es un texto que tiene rasgos tanto de la "estética del exilio" como de la "estética migrante". Se trata de una novela que si bien parece romper con muchas propuestas estéticas y lógicas de representación de las anteriores generaciones de escritores caribeños, en realidad, constituye una suerte de eslabón intermediario entre ambas estéticas, las que por otra parte es difícil que se realicen en forma pura y claramente diferenciable. La lectura de la obra de Kincaid - y, en este caso particular, de Lucy-- muestra que muchos de los problemas que preocupaban a los escritores de la primera generación en relación a su isla de origen siguen estando presentes y convocan, también, a las autoras emigradas a Estados Unidos. A partir del análisis de la crítica que hace Kincaid a la educación colonial recibida en su isla de Antigua, me interesa mostrar que uno de los problemas que puede tener la categoría de "literatura migrante" es la de negar la especificidad de la historia de regiones y países que hasta hace muy poco han sido colonias. Por ejemplo, los cuestionamientos de Kincaid a la educación colonial recibida en Antigua, centrada en gran medida en el rechazo a la imposición del canon británico, muestra la relevancia que siguen teniendo en las narrativas contemporáneas las particulares coordenadas políticas y culturales del lugar de origen. Los límites a la desterritorialización y a la difuminación de fronteras que propone Mardorossian, como características de las literaturas más "actuales", provienen de las particularidades históricas y difícilmente "intercambiables" de los territorios de origen de los migrantes. No se trata de desconocer que existen nuevas propuestas estéticas y de representación en la literatura de mujeres de la diáspora caribeña, sino de comprender en qué medida las concepciones y visiones transnacionales entran en tensión o resuenan con problemáticas nacionales y regionales del Caribe.

Lucy puede ser leída como una "novela de formación de artista" -Künstlerroman - que, si bien retoma muchas concepciones románticas sobre el individuo y el arte, también apela a la particularidad de la experiencia de 
una protagonista que proviene de una colonia caribeña. En Lucy -y este es un rasgo que recorre toda la obra de Kincaid - la reflexión sobre el arte y la producción simbólica está siempre asociada a la consideración de las condiciones materiales de existencia de los sujetos y colectivos que producen y reproducen simbólica y materialmente a sus sociedades.

\section{LA EXPERIENCIA DE LA MIGRACIÓN Y LA FORMACIÓN DE LA ARTISTA}

Al igual que Julia Álvarez en Yo (1997) y Edwidge Danticat en Breath, Eyes, Memory (1994), Jamaica Kincaid construye a la protagonista de Lucy sobre la base de sus vivencias personales. Es por eso que Ian Smith (2002) denomina este texto una "autobiografía ficcional", mientras para María Helena Lima (2002) toda la producción narrativa de Kincaid constituiría un esfuerzo, más acumulativo que lineal, de construcción de un único personaje: el de la escritora.

Lucy, como Kincaid, llega a Estados Unidos - a una ciudad que aun cuando no sea nombrada en forma explícita claramente remite a Nueva Yorkantes de los veinte años a trabajar como au-pair en casa de una familia blanca acomodada. Ahí, Lucy establece una relación especialmente fuerte con Mariah, la madre de las niñas que le toca cuidar. La narradora-protagonista relata sus impresiones y vivencias a lo largo de su primer año de estadía en el nuevo país, en un texto dividido en cinco capítulos que coinciden aproximadamente con cada una de las estaciones del año, comenzando y terminando en un invernal día de enero. El relato, en sus distintos momentos y con contenidos diversos, sigue una lógica similar a lo largo de todo el texto: cada experiencia nueva con la que Lucy se ve confrontada en Estados Unidos es puesta en relación con alguna historia parecida (o por lo menos sentida así por la protagonista) vivida por ella o por algún conocido en su isla de origen. Va surgiendo así una textualidad construida sobre la base de contrapuntos entre las experiencias "en la isla" y en la "gran ciudad", que se corresponden a la oposición entre "pasado" y "presente". Esta oposición, establecida en términos temporales, dialoga con una desarrollada en torno a parámetros espaciales: se trata de las relaciones entre "profundidad" y "superficie", muchas veces traducidas en términos de "realidad" y "apariencia", respectivamente. Este eje que podríamos denominar vertical (lo profundo, lo que está abajo versus lo superficial, ubicado en el exterior) estructura la mayor parte de los sueños y reflexiones del primer año de Lucy en Nueva York. Así, cuando dice "en este mundo uno nunca debe asumir que las cosas son lo que parecen" o "todo permanece igual $\mathrm{y}$, sin embargo, nada es igual", Lucy va subvirtiendo su

\footnotetext{
2 Jamaica Kincaid. Lucy. New York: Farrar, Straus and Giroux, 1990, 78, 79. Citaremos por esta edición.
} 


\section{Lucía Stecher Guzmán}

propia autoridad narrativa puesta a demostrar su determinación a romper con un pasado que, contrariamente, es evocado, una y otra vez, en forma obsesiva: "Yo podía ver la identidad en todas las cosas; yo podía ver el presente asumir una forma, la forma de mi pasado" (90). Los enunciados de la narradora, combinados con características estructurales del relato, van difuminando la fuerza de la oposición pasado/isla presente/Nueva York, como ejes que estructuran y permiten significar la experiencia de la migración. Esto no significa que la tensión establecida por estos polos, así como su fuerza interpretativa, no estén presentes en el texto. Se trata de una presencia que va acompañada por las huellas de su propia descomposición.

El primer capítulo de Lucy se detiene sobre lo que significa dejar lo conocido para aventurarse en un mundo nuevo. La narradora se siente desconcertada y afligida por la pérdida de los referentes sensoriales a través de los cuales estaba acostumbrada a habitar su lugar natal. Una vez fuera de éste, empieza a percibir que ni siquiera los colores, los olores, las sensaciones, son inmunes al cambio de país. La mañana siguiente a su llegada, Lucy despierta relativamente contenta al ver que un sol claro brilla y entra por su ventana. Animada, se levanta y se pone un vestido delgado, "el mismo tipo de vestido que me pondría si estuviera en casa a punto de salir a un día de campo" (5). Al poco rato, sin embargo, Lucy se da cuenta de cuán equivocada estaba. En ese lugar, que el sol brillara no significaba que hiciera calor

Yo no sabía que el sol podía brillar y el aire permanecer frío; nunca nadie me lo había dicho. ¿Qué extraño sentimiento! Algo que siempre había sabido — de la misma manera que sabía que mi piel era del color marrón de una nuez frotada repetidamente con un paño suave, o así como conocía mi propio nombre-, algo que daba por sentado, "el sol brilla, el aire es cálido" no era así. Ya no estaba en una zona tropical, y darme cuenta de esto penetró mi vida como un flujo de agua que dividió lo que antes era terreno seco y sólido, creando dos riberas, una de las cuales era mi pasado - tan familiar y predecible que incluso me hacía feliz pensar en mi infelicidad de antes- y el otro mi futuro, una ribera gris, una marina nublada y lluviosa sin botes a la vista. Ya no estaba en una zona tropical y sentía frío dentro y fuera, la primera vez que tenía una sensación así (5-6).

Ya en este primer capítulo, Jamaica Kincaid expresa muy bien las dimensiones más cotidianas y sensibles de la experiencia migratoria. El cambio de entorno conlleva la interrogación de las certezas más profundas, de aquellos saberes que guían inconscientemente muchas de nuestras conductas y decisiones cotidianas. Sólo fuera del lugar de origen, Lucy puede ver cuestionada lo que consideraba una relación de causalidad incontestable: que cuando el sol sale el aire es tibio. La pérdida de esas certezas básicas hace que 
la protagonista sienta su vida profundamente dividida entre el pasado en su isla natal y el presente/futuro en un lugar frío y gris.

La forma como se describe el encuentro de Lucy con el mundo nuevo que la rodea en Nueva York configura un momento central en su proceso de desarrollo como artista. En su traslado desde Antigua a Nueva York cumple acá con el rol que para los formalistas rusos tenía el arte: producir extrañamiento, desautomatizar las percepciones. Lucy descubre un mundo de percepciones y de asociaciones sensoriales que son nuevas para ella, lo que la lleva a desarrollar una mirada atenta a todo lo que la rodea.

El juego de la extrañeza y la diferencia se articula pronto con la posibilidad que descubre Lucy de reconocer una serie de continuidades entre su vida y sus relaciones en su isla natal y la que está empezando a vivir en Estados Unidos. Su perspectiva de afuerina y su aguda perspicacia le permiten distinguir en su nuevo entorno las grietas casi imperceptibles que amenazan la integridad de las construcciones aparentemente perfectas que la rodean. Así, ella es la primera en darse cuenta del desamor creciente de Lewis hacia Mariah, a quien finalmente deja por su mejor amiga. En este caso, las lecciones aprendidas en la isla no parecen ser inadecuadas para interpretar la realidad, aparentemente más sofisticada, de las relaciones en Nueva York: "en los hombres no se puede confiar" (80). En algunos casos las semejanzas superficiales se asocian a diferencias de fondo; otras veces lo aparente son las diferencias y en lo profundo aparecen las similitudes. Siempre, lo importante es aprender a desarrollar miradas caleidoscópicas, capaces de aprehender la realidad poniendo en relación sus distintos niveles. ${ }^{3}$

\section{EL EPISODIO DE LOS NARCISOS}

En el segundo capítulo, titulado "La lengua", Lucy muestra cómo mientras para Mariah la belleza de los narcisos, asociados a la llegada de la primavera, es indiscutible para alguien que proviene del Caribe anglófono se trata de flores que despiertan sentimientos conflictivos. La crítica postcolonial se refiere a esto como la Daffodil gap — brecha de los narcisos- que da cuenta del abismo existente entre las lecturas y la formación estética entregada por la administración británica a los colonizados, y las experiencias de éstos con la naturaleza y la geografía que efectivamente los rodeaba.

\footnotetext{
${ }^{3}$ Además del desarrollo individual de una sensibilidad que la acerca al mundo de los artistas con el que Lucy se siente cada vez más identificada, es posible reconocer en sus aproximaciones y descripciones de la vida norteamericana una suerte de esfuerzo etnográfico, que constituiría una inversión de la mirada etnográfica tradicional. En lugar del sujeto occidental que viaja y retrata la vida de tribus y poblaciones apartadas, Lucy se dirige al corazón mismo del mundo moderno e intenta comprender cómo es que los sujetos y sus relaciones llegan a ser lo que ella observa y que no considera, en absoluto, "natural".
} 


\section{Lucía Stecher Guzmán}

Lucy tuvo que leer y memorizar en su escuela de Antigua el poema "I Wandered Lonely as a Cloud" (más conocido como "The Daffodils" o "Los narcisos") del poeta romántico inglés William Wordsworth. Ya en Estados Unidos, Lucy es invitada por Mariah a visitar un campo de narcisos apenas llegue la primavera. Ante esto, Lucy le cuenta que de niña había sido elegida para recitar el poema de Wordsworth ante un auditorio de padres, profesores y compañeros. Si bien había sido felicitada por su presentación, Lucy se había prometido olvidar cada palabra de ese poema, debido principalmente a la sensación de falsedad e impostura que la invadió durante su aparición pública. $\mathrm{Su}$ rechazo fue tal que esa misma noche había tenido una pesadilla en la que era perseguida y finalmente aplastada por "ramos y ramos de los mismos narcisos que había jurado olvidar" (18). Claramente, Lucy sintió que su integridad era amenazada por esas flores ajenas e impuestas, a las que tuvo que rendir homenaje aun sin conocerlas. La fuerza de la imposición colonial es tal que la protagonista, en su sueño, fue literalmente aplastada por las flores foráneas, bajo las cuales terminó por desaparecer. Es decir, percibió como niña el peligro de una educación que buscaba borrarla como antillana para, en su lugar, intentar construir una mala imitación del modelo inglés. ${ }^{4}$

El episodio de los narcisos no termina ahí. Poco después, cuando efectivamente llega la primavera, Mariah decide llevar a Lucy a ver un campo de narcisos. Piensa que ante la visión de la belleza de las flores, Lucy tendrá que reconciliarse con ellas. Pero, aun sin saber qué flores son las que Mariah le señala con tanto entusiasmo, lo primero que siente Lucy es un deseo irrefrenable de matarlas, de cortarlas y arrancarlas de raíz. La emoción de Mariah al mostrarle a Lucy algo bello, que pudiera hacerle olvidar el malestar por el poema, rápidamente se transforma en desconcierto cuando la escucha decir: "¿Mariah, te das cuenta que a los diez años tuve que aprender de memoria un poema sobre unas flores que no vería en realidad hasta que tuviera diecinueve años?" (30). Lo que no ve Mariah, porque no forma parte de su experiencia de vida, es que pese a su forma bella e inocente, los narcisos fueron -para Lucy y para muchos otros niños - un instrumento más de dominación imperial. A través de la poesía aprendida en la infancia, niños y niñas aprenden a admirar la belleza de unas flores que no conocen, en perjuicio de aquellas que ven cotidianamente, pero que no entran dentro del "canon" de lo bello y poético. Como señala Joseph (2002), este encuentro de

\footnotetext{
${ }^{4}$ En una de las numerosas entrevistas que ha concedido, Jamaica Kincaid se refiere a su educación en los siguientes términos: "Yo memoricé a Wordsworth cuando era una niña, a Keats, todo tipo de cosas. Era un intento por convertirme en un cierto tipo de persona, el tipo de persona para el que, en cualquier caso, ellos no tenían ningún lugar. Una persona negra educada. Fui llenada de un montón de cosas, así que terminé por usarlas” (Bonetti, 1992:130).
} 
Lucy con los narcisos da cuenta del doble desplazamiento de los referentes reales de las construcciones simbólicas con las que se educa a los sujetos colonizados: Lucy debe esperar a trasladarse tanto temporal como espacialmente para encontrarse con las flores a las que celebró en la infancia.

La confrontación entre Mariah y Lucy en torno a los narcisos muestra cómo mientras para la primera existe continuidad entre formación estética y experiencia vital, en el caso de Lucy ambas están profundamente divorciadas. Con una formación literaria centrada en el canon inglés, Lucy resiente la inadecuación entre los contenidos representados en los textos leídos en la escuela y el entorno caribeño de sus experiencias infantiles. Esto refuerza la convicción de la protagonista de Kincaid de que es necesario revisar todos los saberes y percepciones heredadas, las que invisibilizan las relaciones de poder que las sustentan y que hacen parecer como naturales.

\section{MODELOS (IM)POSIBLES: ENCUENTROS CON PAUL GAUGUIN}

En sus esfuerzos por romper con el pasado de la isla $-\mathrm{y}$ de liberarse del poder de una madre descrita como posesiva y avasalladora $-{ }^{5}$ Lucy va buscando modelos con los cuales identificarse. Una vez más, es Mariah quien le abre una puerta que parece promisoria: la lleva a un museo y le presenta la obra de Paul Gauguin. Más que referirse a su pintura o identificarse con los sujetos y mundos representados en ella, Lucy se detiene a reflexionar sobre la trayectoria del pintor

Había sido un banquero que tenía una vida cómoda con su mujer y sus niños, pero eso no lo hacía feliz; entonces los abandonó y fue al rincón opuesto del mundo, donde era más feliz. (...) Inmediatamente me identifiqué con los anhelos de ese hombre; comprendía la sensación de encontrar que el lugar en que naciste es una prisión insoportable y de querer algo completamente distinto de lo que nos es familiar (...) (95).

Se despliega acá en pleno el tópico del artista que necesita dejar atrás sus comodidades y su burguesa vida familiar para realizarse en el arte. Lucy siente que puede estar cerca de Gauguin. Pero luego reconoce que sus caminos son opuestos. Y eso tiene sus costos: Gauguin se desplaza del centro (Francia) al margen (Tahiti); el personaje de Kincaid, por el contrario, busca un lugar en el centro del mundo neocolonial (Nueva York) mientras procede de una colonia (Antigua). No son trayectorias iguales, la inversión de la dirección del desplazamiento no implica equivalencia alguna. En este sentido

\footnotetext{
${ }^{5}$ La conflictiva relación de Lucy con su madre, y los esfuerzos que realiza por separarse emocionalmente de ella, constituyen otro de los ejes importantes del relato de su primer año en Nueva York. Dado que ha sido uno de los temas de la obra de Kincaid más estudiados, decidimos no profundizar en él. Cfr. Covi, 2003; Ferguson, 1994.
} 


\section{Lucía Stecher Guzmán}

podríamos decir que Kincaid se aparta de la indeterminación territorial que según Mardorossian caracteriza a la estética migrante. En esta narrativa es posible reconocer la persistencia de las relaciones entre un centro hegemónico y periferias que dependen tanto simbólica como materialmente de las relaciones con el primero.

Además, está la diferencia de género, otro factor que Lucy siente que la distancia de Gauguin. Mientras la del pintor es una vida que puede ser encontrada en los libros ("acababa de empezar a darme cuenta que la vida de los hombres siempre está en los libros", 95) ella es "una joven mujer proveniente de los márgenes del mundo, y cuando dejé mi hogar tenía amarrado alrededor de mis hombros el manto de una sirvienta" (95). Sin embargo, reconocer lo que la distancia de un pintor como Gauguin, a la vez que darse cuenta de que el mundo de los artistas es básicamente masculino, no le impide decidir que quiere ser una de ellos, que quiere estar para siempre "con la gente que permanece aparte" (98).

Lucy recorre entonces, en su primer año de emigración, un camino que le permite ir reconociendo todo lo que ella no es y todo lo que no quiere ser. Más que la mirada que le devuelven los otros - factor que en muchas otras novelas de migrantes es fundamental en el reconocimiento de identidades raciales y étnicas negadas o silenciadas en el país de origen- en el caso de Lucy son los contrapuntos que establecen internamente los que le van mostrando las determinaciones que la distinguen de los demás. Reconoce en primer lugar las diferencias que la separan de Mariah, con relación a quien se pregunta permanentemente: "¿Cómo puede alguien llegar a ser de esa manera?". La cuestión está, principalmente, en el pensamiento feminista blanco de Mariah que, en toda su bondad, parece ciego a las diferencias de clase y raza que la separan de Lucy. Mariah es un personaje que se desenvuelve en un mundo de continuidades estéticas y experienciales por lo que nunca se ve compelida a cuestionar su propia formación y su posición privilegiada en la sociedad.

Sin embargo, y a pesar de todos los cuestionamientos y críticas que plantea el personaje de Kincaid a la sociedad occidental y al modelo de sujeto privilegiado que se constituye en su seno (Mariah, Gauguin) la descripción que hace Lucy de su formación como artista se sustenta principalmente sobre modelos románticos de individuación. Lucy interpreta sus transformaciones y su desarrollo como procesos internos, que no responden en primera instancia a la imagen que los "otros" le devuelven de sí misma ni a las experiencias que va teniendo en términos sociales y afectivos. En el último capítulo significativamente titulado "Lucy", la narradora-protagonista se encuentra en una situación muy distinta a la de un año atrás: ha dejado de trabajar como $a u$ pair, comparte un departamento con su amiga Peggy, de quien se ha ido 
distanciando progresivamente, tiene a un artista como amante - del que también se aleja-y, lo más importante, tiene una cámara fotográfica y un trabajo como ayudante de un fotógrafo profesional. Si bien Lucy sigue sintiéndose sola y conserva la rabia que la acompaña a lo largo de la novela, tiene mucha más confianza en su poder de construirse a sí misma

(...) las cosas que no podía ver de mí misma, las cosas sobre las que no podía poner mis manos -esas cosas habían cambiado y yo todavía no las conocía bien. Yo comprendí que me estaba inventando a mí misma, y que lo estaba haciendo a la manera de un artista más que de un científico. No podía contar con la precisión o el cálculo; sólo podía contar con la intuición. No tenía nada preciso en mente, pero cuado el cuadro estuviera completo sabría. No tenía una posición, no tenía dinero para mí. Tenía mi memoria, tenía rabia, tenía desesperación (134. Énfasis mío).

Lucy concibe la soledad y el aislamiento como condiciones indispensables, aunque dolorosas, para poder "construirse a sí misma" sin correr el riesgo de adaptarse a roles y determinaciones impuestas por la sociedad y por las personas que la rodean. Es de alguna manera una encarnación contemporánea de la figura decimonónica del artista atormentado que debe dejar atrás la sociedad que lo constriñe para realizarse en su arte. Hay, en los propósitos de autoinvención de Lucy, mucho de la confianza del liberalismo moderno en las capacidades de los individuos por construir, con esfuerzo y dedicación, su propio destino. ¿Y qué mejor lugar para desplegar esa confianza que los Estados Unidos, la tierra de las oportunidades y de los sujetos que se hacen a sí mismos? Dentro de toda su ironía y ácida crítica a los valores de la sociedad norteamericana, Kincaid construye en esta novela un personaje que en alguna medida sigue los mandatos culturales norteamericanos de auto-realización, aunque sea posicionándose en términos críticos y contra-culturales. Obviamente sería muy burdo leer Lucy dentro del paradigma del logro del "sueño americano". Se trata, por el contrario, de un texto que, tras la aparente simplicidad de su prosa, articula diversos modelos y narrativas que pueden aparecer como muy distintos y contradictorios. Una de ellas es la referida recientemente y que pone a Lucy en relación con los discursos de subjetivación y autonomía de la modernidad liberal y el romanticismo, los que, a su vez, participan también de la configuración de las narrativas modernistas y de la estética del exilio. Claro que Lucy realiza este recorrido desde un particular y marginal posicionamiento en términos de género, raza y clase.

\section{A MODO DE CONCLUSIÓN}

Uno de los rasgos característicos de la estética del exilio es el rol que se le concede a la posibilidad o fantasía de retornar a la tierra natal. En este 


\section{Lucía Stecher Guzmán}

sentido, Jamaica Kincaid se aparta de esta estética y asume, más bien, las características que Mardorossian le atribuye a la literatura migrante. Lucy puede, también, ser leída como un proyecto deconstructivo de los discursos que idealizan el retorno, la reintegración al colectivo de origen y el recurso obsesivo a la importancia de la memoria y el pasado. Este esfuerzo de deconstrucción, sin embargo, está atravesado, a su vez, por tensiones y contradicciones que evidencian el carácter complejo y polisémico de la obra de Kincaid. Con respecto al estatus acordado a los recuerdos y a las revisiones del pasado en Lucy, cabe destacar que mientras la narradora-protagonista manifiesta abiertamente su rechazo hacia las narrativas de la nostalgia, la idealización de la tierra natal y la posibilidad del retorno, el relato de su vida se estructura en términos de una obsesiva revisión del pasado. La autora implícita o conciencia autorial introduce así una relación de tensión entre las afirmaciones y reacciones de la narradora — que manifiesta su aspiración a liberarse de todas las herencias con las que carga desde su infancia- y una estructuración del material narrativo en términos autobiográficos, orientada hacia la reconstrucción de una historia personal. Las contradicciones entre la necesidad de recordar y olvidar, de separarse y no perder la conexión, se hacen especialmente evidentes en términos de la relación de Lucy con su madre. Mientras más enfáticamente manifiesta su deseo de separarse de la figura materna, más fuerte resulta la necesidad de recordarla y de, a partir de este ejercicio, desarrollar estrategias para conjurar su poder (Stecher, 2006).

La escritura de Kincaid cuestiona que la reintegración a la tierra natal sea un momento realmente deseable, sobre todo, en la medida en que es asociada a un retorno al espacio maternal. Este escepticismo está fundado en la homologación que se produce entre la figura materna y el poder colonial, que aparecen como perpetuadores cómplices de una estructuración social patriarcal y eurocéntrica. Así, en Lucy la necesidad de mantenerse a distancia de la madre no está motivada únicamente por su historia personal — por más intensa y dolorosa que ésta aparezca - sino que tiene, igualmente, una importante connotación política. Separarse de la madre es necesario, también, para liberarse de la herencia colonial que ha buscado ejercer poder y control sobre su forma de pensar y de vivir su cuerpo y sexualidad, pero, una vez más, la liberación o el olvido sólo parecen posibles a través de un ejercicio radical de reconstrucción de la memoria. El episodio de los narcisos ejemplifica la rabia de Lucy frente a una educación en el marco de una ideología colonial empeñada en convertirla en la copia mal hecha de una dama inglesa. En Lucy, a diferencia de otras novelas que reconstruyen la experiencia de la migración o el exilio, los dolores más fuertes no están asociados al abandono de la tierra natal, sino que llevan la marca de la situación colonial a la que ésta se encuentra sometida. 
Entre "los placeres del exilio" y los descontentos de la migración

\author{
Universidad Alberto Hurtado* \\ Facultad de Filosofía y Humanidades \\ Departamento de Lengua y Literatura \\ Alameda Bernardo O'Higgins1869, Santiago (Chile) \\ lstecher@uahurtado.cl
}

\title{
BIBLIOGRAFÍA
}

ALVAREZ, Julia. Yo. New York: Plume, 1997.

BONETTI, Kay. “An Interview with Jamaica Kincaid”, en The Missouri Review 15.2. (1992):125-142. Columbia: University of Missouri.

COVI, Giovanna. Jamaica Kincaid's Prismatic Subjects. Making Sense of Being in the World. London: Mango Publishing, 2003.

DANTICAT, Edwidge. Breath, Eyes, Memory. New York: Vintage Books, 1994.

EDMONDSON, Belinda. Making Men: Gender, Literary Authority, and Women's Writing in Caribbean Narrative. Durham: Duke University Press, 1999.

FERGUSON, Moira. Jamaica Kincaid: Where the Land Meets the Body. Charlottesville: University Press of Virginia, 1994.

JOSEPH, Betty. "Gendering Time in Globalization: The Belatedness of the Other Woman and Jamaica Kincaid's Lucy", en Tulsa Studies in Women's Literature. Vol. 21, No 1 (Spring, 2002):67-83. Tulsa: University of Tulsa.

JURNEY, Florence. Representations of the Island in Caribbean Literature: Caribbean Women Redefine Their Homelands. Lewiston - Queenston- Lampeter: The Edwin Mellen Press, 2009.

KINCAID, Jamaica. Lucy. New York: Farrar, Straus and Giroux, 1990.

LAMMING, George. The Pleasures of Exile. United States of America: The University of Michigan Press. 1992.

LIMA, María Helena. "Imaginary Homelands in Jamaica Kincaid's Narratives of Development", en Callaloo 25.3 (2002):857-867.

LOW, Gail. "Finding the Centre: Publishing Commonwealth Writing in London - The Case of Anglophone Caribbean Writing 1950-65", Journal of Commonwealth Literature, 37:2. (2002):21-38.

MARDOROSSIAN, Carine. "From Literature of Exile to Migrant Literature". Modern Language Studies, 32. 2. (Autumn, 2002):15-33.

SAID, Edward. Reflections on Exile and Other Essays. Cambridge: Harvard University Press, 2003.

SMITH, Ian. "Misusing Canonical Intertexts: Jamaica Kincaid, Wordsworth and Colonialism's absent things", en Callaloo 25. 3. (2002):801-820.

STECHER, Lucía. "Salir del país natal para poder regresar: desplazamientos y búsquedas identitarias en la escritura de mujeres caribeñas contemporáneas". Tesis doctoral, inédita. Universidad de Chile, 2006.

SUGG, Katherine. "I would rather be dead": Nostalgia and Narrative in Jamaica Kincaid's Lucy", en Narrative, 10.2. (May 2002):156-173. 